\title{
A qualitative study of urban hospital transitional care
}

\author{
Joe Feinglass, ${ }^{1}$ Samuel Wein, ${ }^{2}$ Caroline Teter, ${ }^{3}$ Christine Schaeffer, ${ }^{3}$ Angela Rogers ${ }^{3}$ \\ ${ }^{1}$ Division of General Internal Medicine and Geriatrics, Northwestern University Feinberg School of Medicine; ${ }^{2}$ Behavioral Research \\ Associate, Lurie Children's Hospital; ${ }^{3}$ Northwestern Medicine Group Transitional Care, Chicago, Illinois, USA
}

\begin{abstract}
This study is part of a mixed methods evaluation of a large urban medical center transitional care practice (NMG-TC). The NMG-TC provides integrated physical and behavioral health care for high need patients referred from the hospital emergency department or inpatient units and who lack a usual source of primary care. The study was designed for internal quality improvement and sought to evaluate staff perceptions of successful transitions for their medically and socially complex patients, and alternatively, the obstacles most likely to negatively impact patient outcomes. All 16 NMG-TC patient care staff were interviewed in a collaborative effort to produce empowered testimony that might go beyond expected clinical narratives. The interview schedule included questions on risk stratification, integrated mental health care, provider to provider handoffs, and how staff deal with key social determinates of patients' health. The constant comparative method was used to deductively derive themes reflecting key domains of transitional care practice. Seven themes emerged: i) the need to quickly assess patient complexity; ii) emphasizing caring for major mental health and substance use issues; iii) obstacles to care for uninsured, often undocumented patients; iv) the intractability of homelessness; v) expertise in advancing patients' health literacy, engagement and activation; vi) fragmented handoffs from hospital care and vii) to primary care in the community. Respondent stories emphasized methods of nurturing patients' self-efficacy in a very challenging urban health environment. Findings will be used to conceptualize pragmatic, potentially high-impact transitional care quality improvement initiatives capable of better addressing frequent hospital use.
\end{abstract}

\section{Introduction}

In December 2011, our large urban academic medical center established a specialized, outpatient practice transitional care clinic, the Northwestern Medicine Group

Correspondence: Joe Feinglass, Division of General Internal Medicine and Geriatrics, Northwestern University Feinberg School of Medicine, 750 N. Lakeshore Dr. 10th Floor, Chicago, Illinois 60611, USA.

Tel.: 312.503.6443 - Fax: 312.503.2755.

E-mail: j-feinglass@northwestern.edu

Key words: Transitional care; Behavioral health care; Hospital use; Patient complexity.

Contributions: the authors contributed equally.

Conflict of interest: the authors declare no potential conflict of interest.

Funding: the study was funded in part by a grant from the Otho S.A. Sprague Memorial Institute.

Received for publication: 5 December 2017

Revision received: 25 April 2018.

Accepted for publication: 25 April 2018

This work is licensed under a Creative Commons Attribution NonCommercial 4.0 License (CC BY-NC 4.0).

CC Copyright J. Feinglass et al., 2018

Licensee PAGEPress, Italy

Qualitative Research in Medicine \& Healthcare 2018; 2:94-100

doi:10.4081/qrmh.2018.7216
Transitional Care (NMG-TC), devoted to post-hospital care for patients discharged from the emergency department (ED), observation care or inpatient units. ${ }^{1}$ Inpatient hospital clinicians were advised to refer patients to the NMG-TC who lacked a regular source of primary care, were uninsured or who seemed overwhelmed by a new, complex or worsening medical condition. The NMG-TC's mission is to achieve a safe and practical transition to primary care medical homes, usually at Federally Qualified Health Center (FQHC) community partners. The NMGTC is located across the street from the hospital, and all appointments are from hospital referrals.

Transitional care is a relatively new medical sub-discipline, and is growing nation-wide as hospitals become interested in savings as well as revenues and are increasingly willing to deploy population-based approaches to care coordination for high risk, high cost patients. Table 1 describes the NMG-TC patient population $(n=4,169)$ through 2017 based on data from each patients' first NMG-TC visit. The NMG-TC primarily sees younger Medicaid and uninsured patients who don't have a usual source of care or a primary care physician, with a large and growing proportion with mental health and substance use diagnoses.

A randomized controlled trial was recently completed and will compare post-referral hospital use for several hundred patients electronically selected to be referred or not referred to the NMG-TC (ClinicalTrials.gov Identifier: NCT03066492). A recently published observational study of over 3,300 patients seen between 2011 and 2016 found a declining proportion of NMG-TC patients with any $90-$ day post-NMG-TC visit hospital use, with an approximate 
$35 \%$ reduction in the likelihood of any 90 -day hospital use between 2011-2012 and 2015-2016. ${ }^{2}$ This decline occurred while the NMG-TC gained increased resources, including integrated mental health care services, many patients gained Medicaid or Health Exchange insurance coverage through the Affordable Care Act, and an increasingly experienced NMG-TC staff developed stronger and more reliable ties with community partners.

This investigation, based on 16 in depth NMG-TC staff interviews in 2016, invited staff to construct narratives about their experiences with their patients' ubiquitous physical, mental and behavioral health challenges. The study was designed by university researchers and staff investigators seeking insights into potential quality of care improvement initiatives. The goal was to collaboratively produce authentic, actively subjective accounts animated by both the joys and deep frustrations of transition care practice.

\section{Materials and Methods}

The interview schedule was designed by NMG-TC clinicians as part of an ongoing, mixed methods evaluation research program. Questions focused on issues prospectively deemed worthy of potential care coordination interventions and with significant quality improvement implications, In particular, the interviews attempted to collaboratively define processing interchanges, or the key moments in the flow of patient care that are most likely to determine patient outcomes. ${ }^{3}$ Study transcription

Table 1. Characteristics of 4169 Northwestern Medicine Group Transitional Care (NMG-TC) Clinic Patients, December, 2011-September, 2017.

\begin{tabular}{lc}
\hline Patient Characteristics at First NMG-TC Visit & Percent \\
\hline Male & 51.8 \\
\hline Female & 48.2 \\
\hline Race and Ethnicity & \\
Non-Hispanic White & 21.4 \\
Non-Hispanic Black & 45.4 \\
Hispanic & 16.8 \\
Other or unknown race and ethnicity & 16.4 \\
\hline Age & \\
18-30 & 23.2 \\
31-49 & 37.0 \\
50-65 & 31.7 \\
65+ & 8.1 \\
\hline Insurance & \\
Private/other & 19.0 \\
Medicaid & 28.0 \\
Uninsured & 45.0 \\
Medicare (including disability) & 8.0 \\
\hline Any ICD coded psychiatric diagnosis & 15.7 \\
\hline ICD International Classification of Diseases. &
\end{tabular}

and analysis was performed independently by the academic health services researchers. All 16 NMG-TC staff were then interviewed and audiotaped by an anthropology-trained research assistant. Interviews were transcribed verbatim. The 12 -item interview schedule was based on sensitizing concepts ${ }^{4}$ that frame staff interactions with patients and allow reflexive coding, including questions about mental health treatment, handoffs between the hospital and primary care or behavioral health medical homes, patient complexity, social service needs assessment and post-transition care follow-up. Interviews were conducted with the general internist and founding medical director, a clinical psychologist, a nurse practitioner, a physician assistant, a registered nurse, two psychiatrists, a clinical psychologist, a clinical pharmacist and three NMG-TC pharmacy residents, a licensed clinical social worker, the operations coordinator, and the three bachelor-degree level Health Advocates who serve as community health workers. To protect confidentiality, all unique professionals were labeled as Clinical Provider below.

Interviews lasted approximately 40 minutes. A constant comparative method was used to derive and code themes with text selection length methods advocated by Kuraski. ${ }^{5}$ Rather than marking fixed, pre-determined idea units, study themes were marked as longer free flowing text segments. After discussing theme definitions, the non-NMG-TC investigators (JF, SW) established interrater reliability for deductive coding of themes at a kappa $>0.90$. The study was ruled exempt by the Northwestern University Institutional Review Board and all participants provided verbal informed consent before being interviewed.

\section{Results}

Interview responses were coded into seven themes, representing how respondents experienced distinct domains of transitional care practice, and their assessments of how each domain was associated with transition outcomes.

\section{The need for rapid risk stratification and patient complexity assessment}

Respondents universally saw their role as providing psychological support and counseling to patients who were all dealing with serious, acute illness that was profoundly disrupting their lives. Respondent narratives emphasized the need to initially categorize complexity so as to quickly assess what level of support services are likely to be required for an ultimately successful transition. They were constantly grappling with how to analyze and break through sometimes chaotic and emotional patient presentations, and which responses seemed to have the highest yield for patient outcomes.

I think these patients are more complex medically and 
socially, and mental health wise, than any other patients that I've ever taken care of. And I've worked internationally, in war zones. I've worked at Cook County Hospital. I worked at FQHCs in Rockford and in Chicago...this is by far the most complex group that I've ever managed. There's chaos in their lives. They have a new medical diagnosis that's either worsened or severe or deadly or something, on top of a complex psychosocial situation. If patients could handle their new cancer diagnosis, they wouldn't be here. If they could handle their new heart failure and new heart attack, new diabetes, new whatever, they wouldn't be here. (Clinical Provider)

...we see a lot of schizophrenic patients. Disorganized, paranoid patients, who are for the most part homeless, and lost to care, because of their illness. (Clinical Provider)

I feel like poverty is almost always playing a role... Lack of social support, lack of resources, and not having a great skill set for managing really, really tough situations... for lack of a better word, just lots of stress. (Clinical Provider)

The NMG-TC social worker described a formal method to stratify patients' baseline risk, to predict each patients' transitional care resources needed at the time of a first visit.

...we implemented a risk-stratified case management protocol that (measures) patient activation (with) twelve questions about the patient's psychosocial functioning... and look at the patient's prior utilization and complexity of illness, and assign them a risk of readmission score. If a patient is uninsured, has a chronic illness and has been lost to care for a year or so, if the patient's like over fortyfive and hasn't gotten their preventive screenings, if transportation is a big barrier...the risk stratification score (is) color-coded. So it's going from cool to hot. Green, yellow, orange, red. Red patients are the most complex. We call them our health advocate patients. Homeless patients... and patients with cancer are automatically assigned to the red group. (Clinical Provider)

NMG-TC providers described evolving care coordination risk models that are perceived to capture psychosocial complexity. These risk assessment techniques provided pragmatic strategies for identifying which patients benefit most from more intensive care including multiple-NMGTC visits or referrals, assignment of Health Advocates for various tasks such as accompanying patients to appointments, and arranging for hospital specialty care.

\section{Challenges to mental health and substance use care}

Staff almost universally emphasized that their patients had huge mental health challenges, whether they had specific psychiatric or substance use conditions or were just in shock from a major physical health decline. Staff frequently noted the advantages of providing integrated mental and physical health care, including access to an internal medicine trained psychiatrist who can manage all aspects of conditions like chronic pain, fibromyalgia, and depression. ....probably the majority of people that show up in our clinic have a behavioral health need. Some kind of reaction to an acute trauma of being shot. Acutely diagnosed with this new heart failure. "I went into the hospital because I was having some chest pain. I come out of the hospital after having a stent. I'm on five medicines, and now I have to retire." Huge, huge issues...they're deer in the headlights I think when they first come in. (Clinical Provider)

A lot of patients ... have had a history of early childhood trauma or poverty or homelessness, often don't always have the best coping skills... (The psychotherapists) both have backgrounds in trauma, which we see a lot of. (Clinical Provider)

An example would be somebody who's got a somatoform disorder, where they believe that something is wrong with their body... sort of the vocabulary that they are using to express their distress. That sort of person can be seen here by a psychiatrist... and they wouldn't rebel against the idea of seeing a psychiatrist. However, if we said to that person, "I'm going to make you an appointment at a mental health clinic," that starts to take them out of their comfort zone. (Psychiatrist)

...you try and figure out what's going to help this person. Is it medications? Or is it more of a situational factor, situational trigger, that's causing the symptoms, and whether you can kind of work with them more one-on-one and just kind of help them get through a crisis, versus it being very chronic and all-enveloping, and you can tell that they have not been functioning for a long time. (Psychiatrist)

Clinic staff often described addressing somatization disorders, the stigma or lack of patient confidence in the effectiveness of mental health treatment, and poor coping skills associated with anxiety disorders.

So the patient might present to the emergency room with chief complaint of feeling blood is dripping down his brain, and there's parasites in his stomach...saying that somebody can help you address some of the stress, or some of the things that are going on in your life, without saying, "You have a mental illness," gets them more willing to engage....And a lot of times, this is severe mental illness. This is schizophrenia, without having any insight, or bipolar disorder. Or it's somebody who has been strong for so long, battling depression or anxiety, and would never go to see a therapist on their own unless a medical provider suggested it. (Clinical Provider)

I've met with patients who don 't have a psych diagnosis, but there is some paranoia, hallucinations, delusions, grandiose...they leave wherever they were living and then just make this impulse decision to move to Chicago. I've had patients who have moved from Michigan and Wisconsin, patients have no support, housing. (Health Advocate)

...both our psychiatrist and the therapists definitely do counseling around cutting back, cutting out, whether it's for opioid addiction or alcohol dependence...(but) addiction is such a devastating disease that I wish I could 
say that we saw wonderful outcomes. It's not that often that we do. (Clinical Provider)

Respondents often noted problems with the availability and effectiveness of alcohol and opioid dependence treatment in Chicago. In particular, staff had to constantly deal with the lack of access to suboxone treatment for heroin users without private insurance, the lack of community mental health homes for homeless patients in need of intensive case management, and extremely long, frustrating social and behavioral services waiting lists, which one provider described as resulting in patients often changing their mind about behavioral health referrals.

\section{Obstacles to care for uninsured and undocumented patients}

A large proportion of NMG-TC patients, even after Affordable Care Act insurance expansions, remain uninsured, including many who still cannot afford Health Exchange plans regardless of the subsidies available. Several staff described the extreme stress of undocumented patients facing complex, burdensome paperwork for free care and the pervasive fear of deportation.

I've had patients where I literally was begging them in the office, "You need to go to the emergency room. This is super dangerous. Your blood pressure is astronomically high. You're at very, very high risk for stroke or a seizure. We really need to get you to go to the hospital now, to safely lower your blood pressure. It's not safe for you to go home now. You could die." And he really, almost refused. It took about a half an hour to get him to finally accept, and one of the biggest things was, because he's uninsured, he was just worried about a huge hospital bill. I think that language plays into that, with our undocumented patients. (Clinical Provider)

NMG-TC staff assist uninsured patients in applying for charity care, prescription assistance, or assist them in applying for insurance coverage. Insurance restrictions described included Medicaid managed care plans that do not cover post-hospitalization transitional care or prior prescriptions just received from inpatient specialists without additional authorizations which may take up to a week or more for approval.

Medicaid managed care plans should cover transitional care. So it makes much more sense to, OK this patient had a heart failure exacerbation, and needs to follow up with cardiology. To be able to even just cover for three months for them to be able to be seen, to kind of finish that work up, or to make sure they're connected, I feel like makes so much more sense, than referring them to an entirely new health system... And then it's just barriers in care and they often fall through the cracks." (Clinical Provider)

\section{Intractable housing uncertainty and social use of the emergency department}

A primary focus of the NMG-TC Health Advocates is to assist patients with social needs, including transportation and social services. Multiple respondents cited housing as the most frequent unmet need.

...patients frequently utilize the ED because it is the only place where they feel listened to and cared for, or perhaps the only place where they can rest in a warm bed for a few hours. (Clinical Provider)

... (some of our) patients are African American men who have been in jail, and they're not even qualified to go to some of these places. Or the lists don't pertain to them. I know we have one patient, he's eighty-one... he's getting progressively worse, because of his heart failure. He just ...needs a place to stay. We've managed to keep him out of the hospital ...for at least a year. And just recently he was admitted, because of a heart failure exacerbation. And that's because he doesn't have housing... I do a lot of wound treatment here too. And a lot of the wounds are from patients who are homeless, who are obese, who have psych issues, personality disorders. Who have nowhere to rest." (Clinical Provider)

Several staff discussed homeless patients reporting that were encouraged by other shelter residents to smoke and drink, were constantly losing their medications, and described the difficulties of psychiatric patients who required medication documentation to get housing.

We've seen patients who went from homeless for ten years, sleeping on the sidewalk, to then getting insurance, getting disability, getting housing....changing the trajectory of their lives that has been the most significant thing that I've seen. When you're trying to talk to your patient who is diabetic, on insulin, has schizophrenia, not on any psych meds, and homeless, trying to talk to him about diet and where they're keeping their insulin and checking their sugar, and everything like that, and then it's like, "Oh, no, I have all this leg swelling. "Without housing, it's so difficult to really make a dent in any of those... We've had a patient who, she's schizoaffective bipolar type, and she has been chronically homeless with intermittent housing. We saw her for several years. But at one point, she was at least stable in an SRO (single room occupancy rental). She wasn't going to the ER a gazillion times. And then, maybe the county sheriffs were going to close down the SRO. And since then, just literally (she) will go from like ER to ER to ER to ER. (Clinical Provider)

Several NMG-TC staff advocated for an ED-based housing program for patients with mental illness who are homeless. These were the patients most often lost to follow-up care, with no ability to manage complex symptoms. The clinic kept some homeless patients' birth certificates in their files to assist with Medicaid annual recertifications.

\section{Expertise in enhancing health literacy, engagement and activation}

Multiple providers described their case management approach as meeting each (patient) where they are at. 
...we do our best to really try to treat patients with respect and kindness and dignity. And for a lot of patients who have been on the outskirts of care for a long time, maybe they haven't seen a doctor in twenty years, and then they go because of hip pain, and they found out they have metastatic prostate cancer. And don't know how to navigate the health system very well, or advocate for themselves very well. So being able to...be present with them...to say, "we have a health advocate who can help you get insurance" or "we have someone who can go to an appointment with you" or, "we have a therapist here, and it sounds like you're having a really tough time, and that sounds like a really normal reaction to everything you're going through."... and to do so kind of quickly, rather than, "Oh, I'll put in a referral for the therapist to, maybe you'll get an appointment in like two months."... to provide them a little sense of hope too, that they're not in this alone. (Clinical Provider)

Many staff respondents defined their transitional care mission as addressing low health literacy and getting their patients to become active and engaged in their care. Reconciling medication regimens for these recently discharged patients was described as a crucial first step.

I've educated on what A1C is with diabetes, or what an INR is with Warfarin, so that they can understand when they're reading results or when their physicians tell them what each of those things means... we kind of don't use medical terminology if we don't have to, just so it's in layman terms. Frequency, when they're taking it each day. Is this morning, noon, after dinner, night time, like "take this with a meal"...we give out pillboxes to patients... that they can fill themselves. (Pharmacy Resident)

Like we had a gentleman who was injecting insulin into his legs when he got Charley horses, because he thought that the insulin would help with his Charley horse. ...this is a patient with mental health comorbidities, so trying to explain to him ...like, "Your insulin is for your blood sugar, and this is where you inject it, and this is how often you inject it." And then he learned that... (Another) patient that has uncontrolled diabetes, and was admitted to the hospital, with blood sugar...in the five hundreds somewhere. I provided ...resources... what does a typical plate look like? How should you divide that up? And what types of foods should you be eating and should you avoid? ...it kind of opened his eyes a little bit, like, "Wait a second, maybe part of this is my responsibility, and that I have to take this on." (Pharmacy Resident)

\section{Fragmented handoffs: to transition care from hospital care}

Respondents frequently described challenges to improving the show rate of patients referred to transition care from the hospital. As the NMG-TC medical director notes below, hospital referral protocols remain informal and can potentially perpetuate inefficient care:

There's not a formal handoff (from the hospital).
Sometimes people will call if they have a really troublesome patient (but) not that often. Sometimes the discharge summary is kind of not super helpful. You end up having to read six or eight different specialist notes... And so a lot of times, something (gets) picked up after they get here, and it has not been documented. So like no one ever noted the patient was having florid psychosis...(Clinical Provider)

I could see how someone who is uninsured and was kind of forced to go into the emergency room because of whatever crisis that they're in...would be very reluctant to come back, when they're concerned about paying for services... (a patient) in there for intoxication or drug overdose, ...that person most likely is going to be scared to come back, and they don't show. (Health Advocate)

Several respondents described the value of Health Advocates who can walk a patient over to the NMG-TC.

...we'll (sometimes) go meet someone in the emergency department or inpatient unit... We call it a warm handoff. ... we think that's been a really effective thing for some of these frequent users of hospital resources. (Clinical Provider)

\section{Fragmented handoffs: from transition care: to primary care in the community}

A majority of respondents echoed the clinic's mantra of stabilizing and then launching patients to on-going primary care as the core mission of the NMG-TC, with a particular sensitivity to mental health issues.

For the patients with mental illness, usually we plan a series of visits around the transition... We talk about what's going to happen. If the patient is working with a health advocate, we will have them go to that visit with the health advocate..., if we're worried about their ability to transition, we will do what we call a soft launch, ... another visit scheduled back with us a week later... and if it didn't stick, they didn't go, we can have another chance. (Clinical Provider)

...we're referring people to places where logistically it works for them, in terms of where they live. I'll sometimes reach out to a provider that I anticipate eventually seeing a patient who I have seen. ...-they give me consent to do it...so that when they come for that first visit, they're actually also meeting behavioral health on that same visit...(Clinical Provider)

NMG-TC staff constantly juggle the clinical and financial disconnects between the academic medical center and FQHC care:

A lot of times, it's the simplest things that fall through, like for example, a patient is doing well with heart failure, on a set regimen. And then they launch to the community and the provider orders...medicine that's not a four dollar generic. It's $\$ 165$... because it got switched to the brand name or whatever. And then the patient just doesn't fill it. And then it's just the beginning of a slippery slope where the disease gets worse, ... and then they get admitted, and 
then we end up with them again. A lot of times, the damage is permanent. (Clinical Provider)

Several respondents also described the fine line between empowering versus enabling patients as potentially undermining subsequent care in the community:

We see patients often who are half hour late, one hour late, two hours late. Walk in. We try to do our best to accommodate. But the reality is like, if you're fifteen minutes late, you're often not seen at other clinics. And probably the receptionists are not making you oatmeal or getting you a bus card or vouchering your medications. (Clinical Provider)

The ease of the clinic is also a detriment. We give them food. Clothes if they need it. Everyone here is so sweet. It's our demise if we don't start setting these boundaries. So, a lot of times, I feel like we don't do the very best job at discharging some of our patients. The ones who are stronger, they can manage. The ones who are not, they don't have family, they don't understand their disease, they are very timid and shy...or they just don't care, those people are the ones who we see again. (Clinical Provider)

I actually had a patient this morning actually cry because she did not want to go to another clinic. She wanted to stay here with us. So I had to explain to her that we don't have any primary care physicians here, and the importance of her having a primary care physician. (Health Advocate)

Almost all respondents lamented the lack of a unified medical record and other follow-up resources, and were unsure of the actual stick rate of discharged NMG-TC patients at FQHC medical homes, a rate that includes some patients lost to follow-up between NMG-TC visits and thus never discharged to care in the community.

\section{Discussion}

Kangovi et al. ${ }^{6}$ interviewed 65 low socioeconomic status patients and described perceptions of their challenges during post-hospital transitions. Themes identified were: i) powerlessness during hospitalization due to illness and socioeconomic factors often related to insurance; ii) misalignment of patient and care team goals as exemplified by a homeless patient being told she needs to weigh herself daily; iii) housing instability leading to ignoring discharge instructions; iv) inability to afford medications or co-pays for follow up visits; v) abandonment by social supports and the health care system after discharge, often associated with failure to communicate questions or problems with medications to hospital providers; vi) patients feeling doomed to fail and deciding not to try. Other studies, also overlapping respondent perceptions from this study, describe stress, lack of transportation, and lack of affordable home care as leading to frequent emergency department (ED) visits and hospitalizations. ${ }^{7-9}$

How well does transitional care address these concerns? Transitional mental health brief intervention pro- grams have been found to successfully prevent psychiatric readmissions and repeat ED use in the US and in Britain. ${ }^{10-14}$ However, the research evidence about the longer term clinical and psychological impact of brief transitional care interventions remains weak. ${ }^{15}$ Revenue focused hospital administrators often continue to see transitional care as a costly, high touch service lacking significant fee-for-service reimbursement.

These study findings suggest otherwise, with respondents uniformly confident about the potential of multidisciplinary, integrated transitional care to bolster patients' self-efficacy in dealing with critical illness and multi-morbidity. Because the study was conducted in the workplace and was explicitly a quality improvement exercise, it is possible that respondents' voices echoed the institutional story and mission of the clinic, rather than their own authentic reflections. Staff authors were blinded to individual respondent identities for the manuscript quotes above but could likely infer who said what. However, as many of the quotes above demonstrate, respondents were consistently able to openly discuss their frustrations with transition care practice, the fragmentation and barriers to care they experienced, and the frequently poor outcomes experienced by their patients.

While many hospital transitional care programs focus primarily on preventing readmissions of older, frail, homebound or end of life patients, ${ }^{16-19}$ the NMG-TC was designed to serve younger, uninsured or underinsured patients with psychosocial and economic barriers to managing their health. The NMG-TC exists in an environment (downtown Chicago) where few if any mental health professionals are willing to see indigent patients. Prior to the NMG-TC, hospital providers were often providing discharged patients with no regular primary care doctor an unreliable FQHC appointment phone number, hoping for the best.

\section{Conclusions}

\section{Using interviews in quality improvement}

These interviews provide a valuable perspective on how highly motivated safety net providers cope with enduring health disparities in access and fragmentation of care while simultaneously struggling with powerful social determinants of urban health. The study interview process embodied a collaborative spirit in which the interviewer's questions were informed by staff investigators, who then engaged staff respondents in creatively constructing thematic narratives. The conversational interview process created multiple layers of meaning in an interactive process. This study demonstrates why interviews are often the best, most efficient, and most meaningful way to shed light on actionable healthcare delivery system change and innovation. ${ }^{20,21}$

The study documents life on the urban safety-net front 
lines from the standpoint of providers who see very complex and vulnerable patients. Study findings are being used to design multiple quality improvement interventions across the domains described above. Examples of current efforts include improved patient risk stratification models that can better prioritize use of clinic personnel and timely care coordination, creation of new mental health homes for patients willing to attend appointments at the medical center, exploring an ED-based housing program in conjunction with community-based housing organizations, designing an electronic master patient index to track appointment outcomes for patients referred to $\mathrm{FQHCs}$, and working with residents and ED physicians to improve the hospital referral process and NMG-TC show rate. In the long run, to dramatically improve outcomes for our highest risk, highest cost patients, there will need to be new transitional care payment mechanisms ${ }^{22}$ and much better integration of home and community-based social services and supports. ${ }^{23,24}$ While these interviews are stories from a single organization, they illustrate many of the challenges common to efforts to implement population-based healthcare practice.

\section{References}

1. Kim CS, Flanders SA. In the Clinic. Transitions of care. Ann Intern Med 2013;158:ITC3-1.

2. Feinglass J, Mallama CA, Rogers A, et al. Using hospital use trends to improve transitional care. Healthc (Amst) 2017.

3. Gubrium JF. Narrative practice and the transformation of interview subjectivity. In: Gubrium JF, Marvasti AB, McKinney KD, eds. The Sage handbook of interview research: the complexity of the craft. Thousand Oaks, CA: Sage; 2012. pp 27-44.

4. Faircloth CA. After the interview: what is left at the end. In: Gubrium JF, Holstein JA, Marvasi AB, McKinney KD, eds. Sage Handbook of qualitative research: the complexity of the craft. Thousand Oaks, CA: Sage; 2012.

5. Kuraski KS. Intercoder reliability for validating conclusions Drawn from open-ended interview data. Field Methods 2000;12:172-94.

6. Kangovi S, Barg FK, Carter T, et al. Challenges faced by patients with low socioeconomic status during the post-hospital transition. J Gen Intern Med 2014;29:283-9.

7. Bergamo C, Juarez-Colunga E, Capp R. Association of mental health disorders and Medicaid with ED admissions for ambulatory care-sensitive condition conditions. Am J Emerg Med 2016;34:820-4.

8. Strunin L, Stone M, Jack B. Understanding rehospitalization risk: can hospital discharge be modified to reduce recurrent hospitalization? J Hosp Med 2007;2:297-304.
9. Kangovi S, Barg FK, Carter T, et al. Understanding why patients of low socioeconomic status prefer hospitals over ambulatory care. Health Aff (Millwood) 2013;32:1196-203.

10. Capp R, Misky GJ, Lindrooth RC, et al. Coordination program reduced acute care use and increased primary care visits among frequent emergency care users. Health Aff (Millwood) 2017;36:1705-11.

11. Hengartner MP, Passalacqua S, Heim G, et al. The post-discharge network coordination programme: a randomized controlled trial to evaluate the efficacy of an intervention aimed at reducing rehospitalizations and improving mental health. Front Psychiatry 2016;7:27.

12. Shaffer SL, Hutchison SL, Ayers AM, et al. Brief critical time intervention to reduce psychiatric rehospitalization. Psychiatr Serv 2015;66:1155-61.

13. Vigod SN, Kurdyak PA, Dennis CL, et al. Transitional interventions to reduce early psychiatric readmissions in adults: systematic review. Br J Psychiatry 2013;202:187-94.

14. Lamanna D, Stergiopoulos V, Durbin J, et al. Promoting continuity of care for homeless adults with unmet health needs: The role of brief interventions. Health Soc Care Community 2018;26:56-64.

15. Billings J, Raven MC. Dispelling an urban legend: frequent emergency department users have substantial burden of disease. Health Aff (Millwood) 2013;32:2099-108.

16. Anderson GF, Ballreich J, Bleich S, et al. Attributes common to programs that successfully treat high-need, high-cost individuals. Am J Manag Care 2015;21:e597-600.

17. Boutwell AE, Johnson MB, Watkins R. Analysis of a social work-based model of transitional care to reduce hospital readmissions: Preliminary data. J Am Geriatr Soc 2016;64:1104-7.

18. Coleman EA, Parry C, Chalmers S, Min SJ. The care transitions intervention: results of a randomized controlled trial. Arch Intern Med 2006;166:1822-8.

19. Golden AG, Tewary S, Dang S, Roos BA. Care management's challenges and opportunities to reduce the rapid rehospitalization of frail community-dwelling older adults. Gerontologist 2010;50:451-8.

20. Mishler EG. The unjust world problem: Towards an ethics of advocacy for healthcare providers and researchers. Commun Med 2004;1:97-104.

21. Mishler EG. Patient stories, narratives of resistance and the ethics of humane care: a la recherche du temps perdu. Health (London) 2005;9:431-51.

22. Bindman AB, Blum JD, Kronick R. Medicare's transitional care payment-a step toward the medical home. N Engl J Med 2013;368:692-4.

23. Windh J, Atkins GL, Simon L, et al. Key components for successful LTSS integration: lessons from five exemplar plans. In: Alliance L-TQ, ed. Project to Develop the business case for LTSS integration: the SCAN Foundation; 2016:1-127.

24. Feinglass J, Norman G, Golden RL, et al. integrating social services and home-based primary care for high-risk patients. Popul Health Manag 2018;21:96-101. 